\title{
AES and XPS characterization of TiN layers formed and modified by ion implantation
}

\author{
V. Melnik, V. Popov, D. Kruger*, O. Oberemok \\ Institute of Semiconductor Physics, NAS of Ukraine \\ 45, prospect Nauki, 252650 Kyiv, Ukraine. Tel/Fax: (38044)265-5724 \\ * Institute of Semiconductor Physics, Walter-Korsing-Str.2, \\ 15230 Frankfurt (Oder) Germany. Tel. (49335)5625-326. Fax. (49335)5625-300
}

\begin{abstract}
Compositional characterization of sputtered and implanted titanium nitride (TiN) layers for microelectronics application is performed based on Auger Electron Spectroscopy (AES) and X-ray induced Photoelectron Spectroscopy (XPS) data. AES shows a strong overlapping of the most intensive peaks of Ti and N. A simple empirical method using intensity relations of Auger spectra is developed for quick estimation of layer composition in small areas. Defined modification of the TiN layers was realized by means of carbon and oxygen implantation to study their influence on quantitative analysis. In difference to standard AES analysis the results of quantification using the method proposed are found to be in good agreement with XPS profiles and with results from Principal Component Analysis (PCA), where peak overlapping is excluded. The influence of oxygen was found to be crucial for standard AES analysis but it could be taken into account in the proposed method. High carbon concentrations show no significant influence on the Ti and $\mathrm{N}$ peak shapes.
\end{abstract}

Keywords: ion implantation, titanium nitride, auger electron spectroscopy, X-Ray photoelectron spectroscopy, component analysis, microelectronics, optoelectronics.

Paper received 17.09.99; revised manuscript received 29.09.99; accepted for publication 11.10.99.

\section{Introduction}

Titanium nitride films are perspective in a wide area of applications, ranging from hard and corrosion resistant coatings [1] to diffusion barrier layers for advanced electronic devices [2-4]. Different formation techniques such as physical or chemical vapor deposition, and magnetron sputtering have been applied. During the last few years the use of ion implantation for the formation of TiN attracts attention [5]. The modification of composition, microstructure and strain in these layers is under investigation to optimize their properties. Therefore, a strong need for laterally resolved quantitative characterization arises.

For large structures in the $10^{2} \mathrm{~mm}^{2}$ range $\mathrm{X}$-ray induced Photoelectron Spectroscopy (XPS) with its high energy resolution allows to separate the peaks of the elements in the spectra and to perform compositional and chemical quantification. However, for analyzing sub-micron areas and small defects in semiconductor metallization structures only Auger Electron Spectroscopy (AES) seems to satisfy the resolution requirements of the SEMATECH Metrology Roadmap [6].

Two main factors hinder the Auger qualitative and quantitative analysis of TiN layers. Firstly, the $\mathrm{Ti}_{\text {LMM }}$ peak overlaps the $\mathrm{N}_{\mathrm{KLL}}$ peak at approximately $387 \mathrm{eV}$ kinetic energy which is the predominant transition of nitrogen. This over- lap is so severe that the peaks are completely superimposed. Secondly the line shape depends on chemical bonding in the Ti compounds, giving rise to additional errors in quantitative analysis. The Target Factor Analysis (TFA) [7] and the method used at Charles Evans Association [8] are sophisticated examples for quantitative analysis. The application of these methods requires the specifically equipment and software, which is not always accessible.

In the given work we present AES and XPS characterization of sputtered TiN films and annealed TiN layers formed and modified by means of high dose ion implantation. A simple empirical method, based on intensity relations of the AES peaks is developed and proposed for a quick estimation of layer composition. Into some of these layers carbon and oxygen (the impurity which usually are found with the analysis) were additionally implanted to study their influence on quantitative analysis. The results of AES quantification are compared with XPS profiles and results from Principal Component Analysis (PCA).

\section{Experimental details}

To investigate the formation of annealed TiN layers we implanted $\mathrm{N}^{+}$ions at room temperature and at $500^{\circ} \mathrm{C}$ into me- 


\section{Melnik et al.: AES and XPS characterization of TiN layers...}

chanically polished Ti samples and sputter deposited Ti layers on Si substrates. An implantation energy $E=100 \mathrm{keV}$ and a dose in the range of $3.8 \cdot 10^{16} \mathrm{~cm}^{-2}$ to $6.5 \cdot 10^{17} \mathrm{~cm}^{-2}$ were chosen. Thin TiN films (30 nm - $100 \mathrm{~nm})$ deposited on Si by means of magnetron sputtering in $\mathrm{N}_{2}$-atmosphere, and TiN/TiSi /Si structures were also used for comparison. AES and XPS depth profiles were measured to determine the layer composition.

The basic Auger analyses were carried out using a $10 \mathrm{keV}, 10 \mathrm{nA}$ electron beam with a beam diameter of about $50 \mathrm{~nm}$ on the «PHI 670» installation. For definition of universality of offered method some part of measurements were carried out on installation 09-ИОС-005 with difffering technical parameters using a $3 \mathrm{keV}, 1 \mathrm{~mA}$ electron beam with a beam diameter of about $10 \mu \mathrm{m}$.

The XPS data were obtained using monochromatic AlKa (1486.6 eV) X-rays. For sputter depth profiling we used a $4 \mathrm{keV} \mathrm{Ar}^{+}$beam rastered over $1.5 \times 1.5 \mathrm{~mm}^{2}$ (PHI670), $2,5 \mathrm{keV} \mathrm{Ar}^{+}$beam rastered over $2 \times 3.5 \mathrm{~mm}^{2}$ (09-OC-005) for AES and over $4 \times 4 \mathrm{~mm}^{2}$ for XPS. Laterally resolved AES measurements were performed to characterize submicron TiN metallization defects. XPS quantification has been performed using sensitivity factors calibrated by means of stoichiometric TiN layers.

\section{Results and discussion}

Fig.1a shows the results of AES peak shape measurements on reference samples of the main Auger transitions of $\mathrm{Ti}$ in $\mathrm{Ti}, \mathrm{TiO}_{x}, \mathrm{TiN}_{y}, \mathrm{TiSi}_{z}$. The most intense peaks are the LMM and LMV transitions near $387 \mathrm{eV}$ and $420 \mathrm{eV}$; intensities of that are designated as $\operatorname{Ti}(1)$ and $\operatorname{Ti}(2)$, respectively. With the emission of valence electrons the LMV line is sensitive to chemical bonding, which results in splitting of the oxide and nitride lines. Changes in peak shape occur also for the LMM line. The indicated spectra can serve as fingerprints for information about composition and phase. Additional information can be extracted from the Ti-LVV peak at $27 \mathrm{eV}$

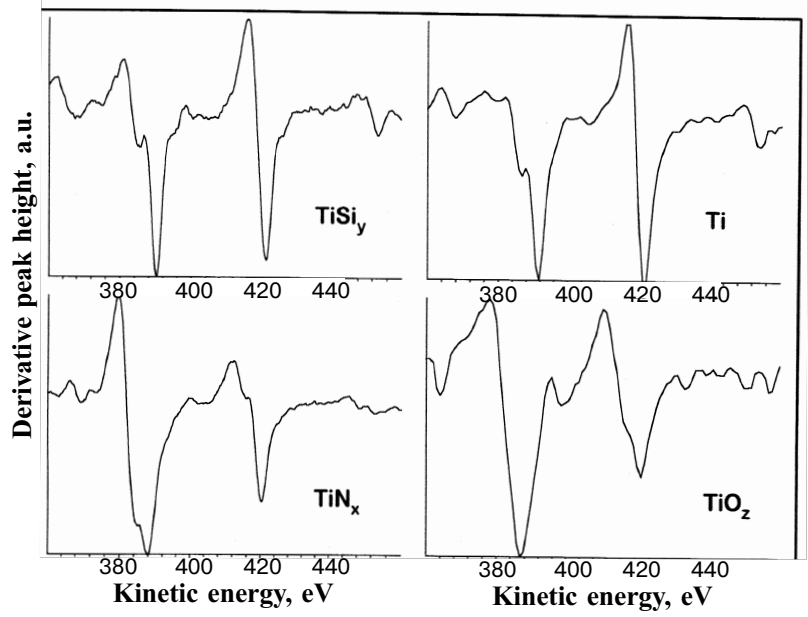

a (not shown) and the Ti-LMM peak at $451 \mathrm{eV}$ which are not influenced by the N-KLL ( $385 \mathrm{eV})$ peak.

The peak-to-peak heights (PPH) of the intense peaks $\mathrm{Ti}(1)$ and $\mathrm{Ti}(2)$, obtained from samples with different composition can be used as a relative and approximate measure of the depth distribution of the elements in the layer. Fig. $1 b$ shows a depth profile through a $\mathrm{TiN}_{\mathrm{x}} / \mathrm{TiSi}_{\mathrm{y}}$ structure, which is oxidized in the near-surface region. Starting from the $\mathrm{Si}$ substrate a plateau region is indicated by the $\mathrm{Si}$ and $\mathrm{Ti}$ intensities. This region is related to the $\mathrm{TiSi}_{\mathrm{y}}$ layer formed by solid-state reaction of $\mathrm{Ti}$ and $\mathrm{Si}$. The next layer contains no $\mathrm{Si}$ and is characterized by an approximately constant peak intensity of $\mathrm{Ti}(2)$ but increasing the $\mathrm{Ti}(1)$ one, which indicats a layer with the nitrogen content increasing towards the surface. The surface layer is oxidized as can be concluded from the oxygen profile and the peak shape analysis (not shown) and is related to $\mathrm{a} \mathrm{TiO}_{z}$ phase. Although, the ratio of the peak intensities $\mathrm{Ti}(1)$ (which is strongly influenced by N-KLL) and $\mathrm{Ti}(2)$ can be used for a rough characterization of different Ti compounds, a simple application of elemental sensitivity factors as in standard quantification techniques yields in incorrect concentrations of such samples. Suitable data processing are required for a more accurate and a quantitative analysis of $\mathrm{TiN}_{x}$ layers, especially of those containing oxygen.

To fabricate samples with different compositions of $\mathrm{TiN}_{x}$, a high dose $\mathrm{N}$ implantation into Ti was performed. A typical depth profile using the peak-to-peak heights (in arbitrary units) for an implantation doses of $6.5 \cdot 10^{17} \mathrm{~cm}^{-2}$ is shown in Fig.2a. Fig.2b shows the result of a TRIM 98 calculation. As it is seen from comparison of Figs $2 a, 2 b$, with increasing $\mathrm{N}$ concentration the Ti(1) intensity increases whereas the $\mathrm{Ti}(2)$ intensity remains almost unchanged. We determined the ratio of the PPH intensities $n=\mathrm{Ti}(2) / \mathrm{Ti}(1)$ for pure $\mathrm{Ti}$ and in our experimental conditions. The intensity relation $\mathrm{Ti}(2) / \mathrm{Ti}(1)$ varies from 1.5 for pure $\mathrm{Ti}$ to 0.5 for TiN. Analyzing the dependence of the intensity relation $\mathrm{Ti}(2) / \mathrm{Ti}(1)$ on nitrogen concentration, the following ex-

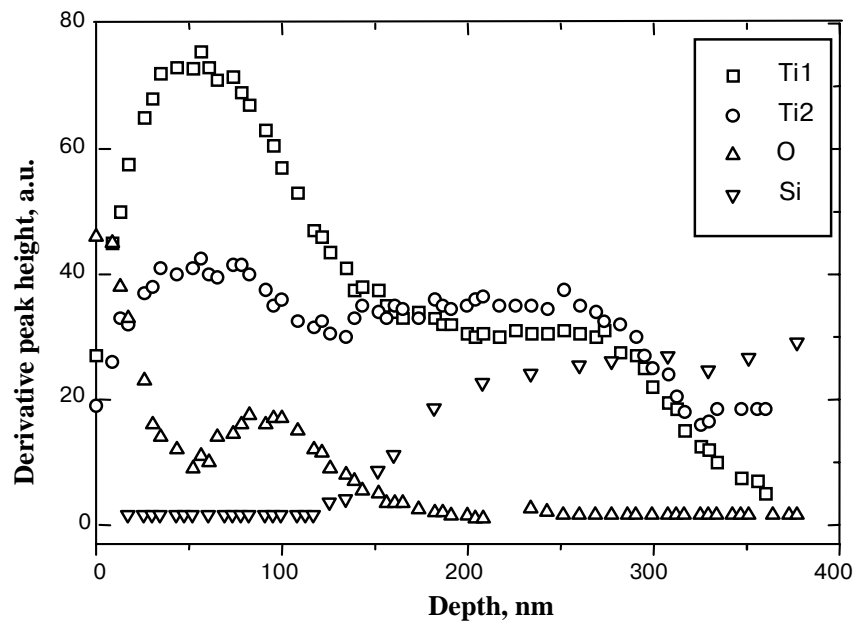

b

Fig.1. (a): Auger line shapes of the Ti-LMM peak for different Ti compounds; (b): Auger depth profile of a TiN/TiSi/Si structure using the peak-to-peak heights of the Si-LVV, Ti-LMM, (overlapping with N-KLL), Ti-LMV, and 0-KLL transitions. 


\section{Melnik et al.: AES and XPS characterization of TiN layers...}

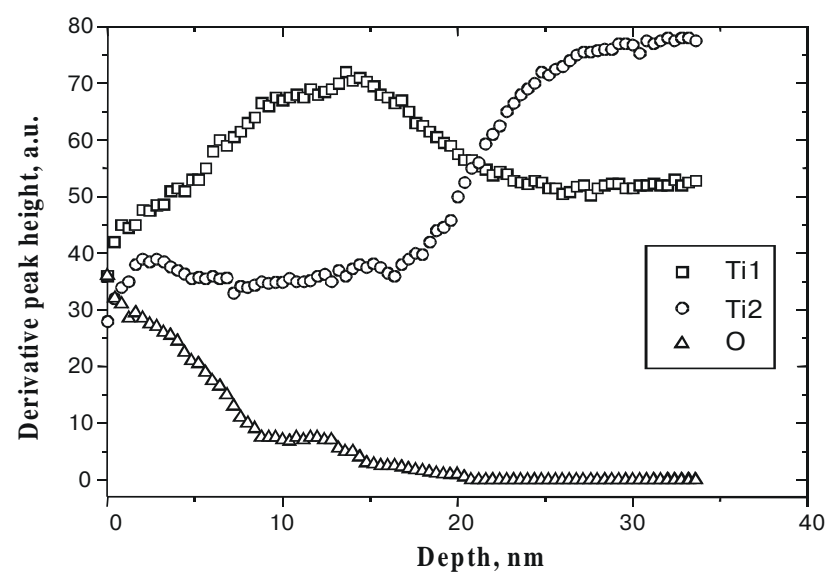

a

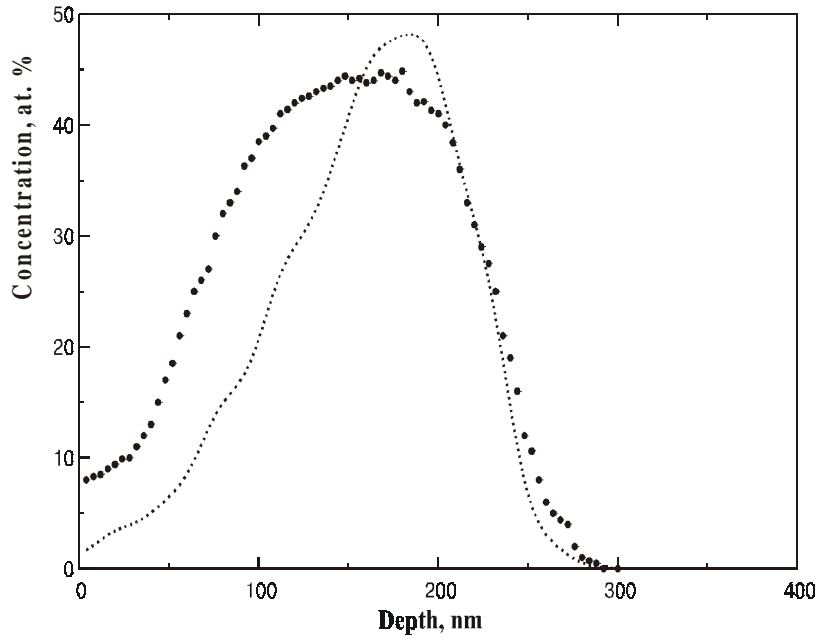

b

Fig.2. (a): Auger depth profile of a $\mathrm{N}$ implanted polished Ti sample $\left(E=100 \mathrm{keV}, D=6 \cdot 10^{17} \mathrm{~cm}^{2}\right)$ using the peak-to-peak heights of the Ti-LMM, (overlapping with N-KLL), Ti-LMV, and 0-KLL transitions; (b): $\mathrm{N}$ concentration in the implanted sample calculated by means of Equ.(1) in comparison to a TRIM 98 calculation.

pression was found empirically for the concentration ratio of $\mathrm{N}$ and $\mathrm{Ti}$ :

$C_{\mathrm{N}} / C_{\mathrm{Ti}}=\{[\alpha \operatorname{Ti}(1)-\operatorname{Ti}(2)] / 2 \operatorname{Ti}(2)\}$

where $\alpha=\operatorname{Ti}(2) / \operatorname{Ti}(1)$ for pure $\mathrm{Ti}$ and is equal to 1.5 for «PHI 670» installation.

Eq. (1) gives a simple relationship for the determination of $\mathrm{N}$ in a $\operatorname{TiN}_{x}$ matrix. Fig. $2 \mathrm{~b}$ shows the $\mathrm{N}$ concentration calculated from the depth profile in Fig.2a using Eq.(1), additionally to the TRIM calculation. A good correlation is obtained for the decreasing part of the profile over a wide area of concentration ranging from about 45 at.\% down to 1 at.\%. However, significant differences occur at the increasing part of the profile near the surface. These differences seem to be mainly related to large amounts of oxygen which influence the ratio of $\operatorname{Ti}(1)$ and $\operatorname{Ti}(2)$.

The influence of oxygen on quantitative analysis was

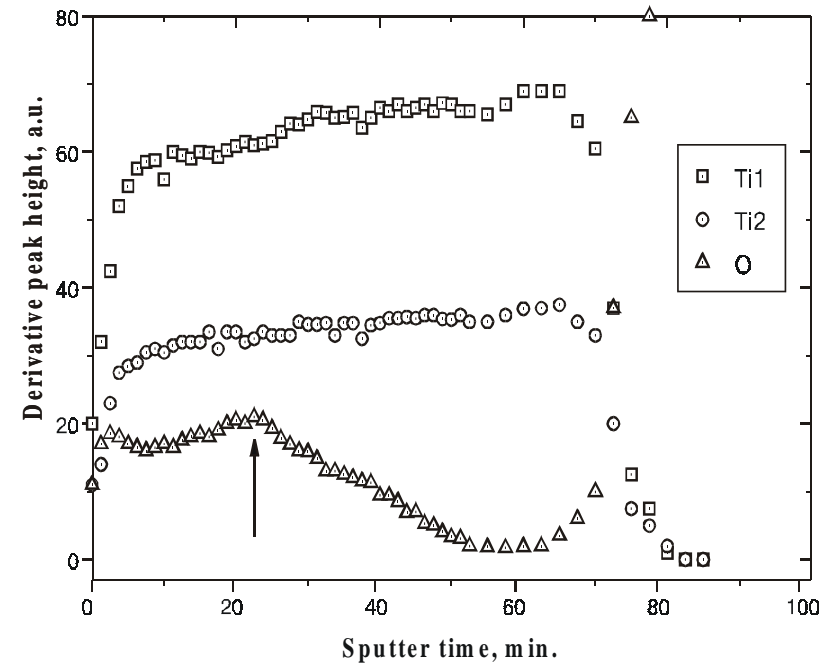

a studied by means of a high dose oxygen implantation into $\mathrm{TIN}_{x}$ layers. Fig.3a shows a corresponding AES depth profile. As seen, the ratio of the PPH intensities Ti(2)/Ti(1) is influenced by the oxygen content. This influence is mainly related to changes in the Ti(1) peak shape (Ti-LMM). Fig.3b shows the influence of oxygen implantation on the spectra. For increasing oxygen concentration by a factor of about 3 , from 6 at. \% to about 20 at.\%, no significant changes in the PPH of the Ti(2) peak can be detected. Therefore, the Ti(2) peak can roughly be used for calculation of the oxygen content in TiN layers. With the knowledge of the oxygen influence Eq.(1) can be used for $\operatorname{TiN}_{x} \mathrm{O}_{y}$ characterization.

Fig.4a presents the results of XPS depth profile of the layer formed by $\mathrm{N}$ implantation into a Ti layer containing about 10 to 15 at.\% of oxygen in the bulk of the layer. Solid line represents the $\mathrm{N}$ concentration calculated using Eq. (1) with the account of the oxygen influence. Starting from this

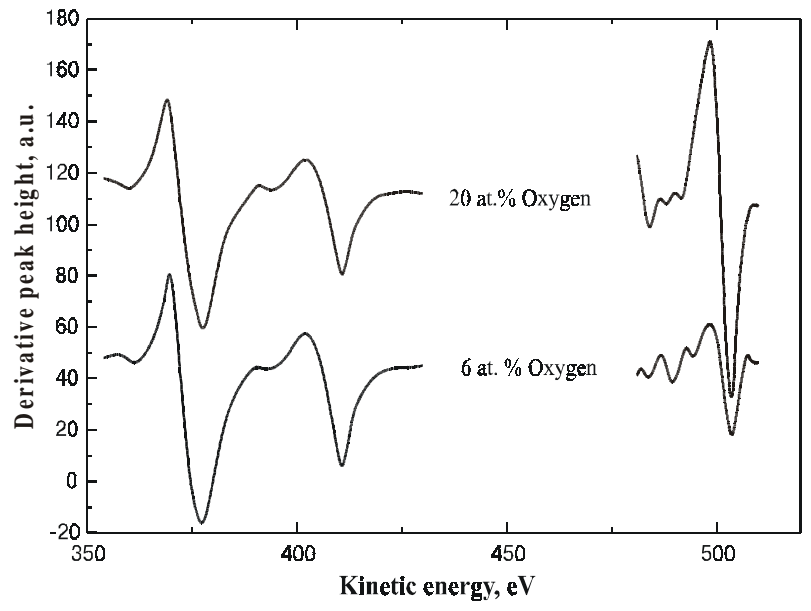

b

Fig.3. Oxygen implantation into a TiN layer on $\mathrm{Si}\left(E=70 \mathrm{keV}, D=6 \cdot 10^{16} \mathrm{~cm}^{-2}\right)$ : (a): PPH - Auger depth profile; (b): line shapes of the Ti-LMM peak for oxygen concentrations of 6 and 20 at.\%. The arrow indicates the $R_{p}$ position for the implanted oxygen. 


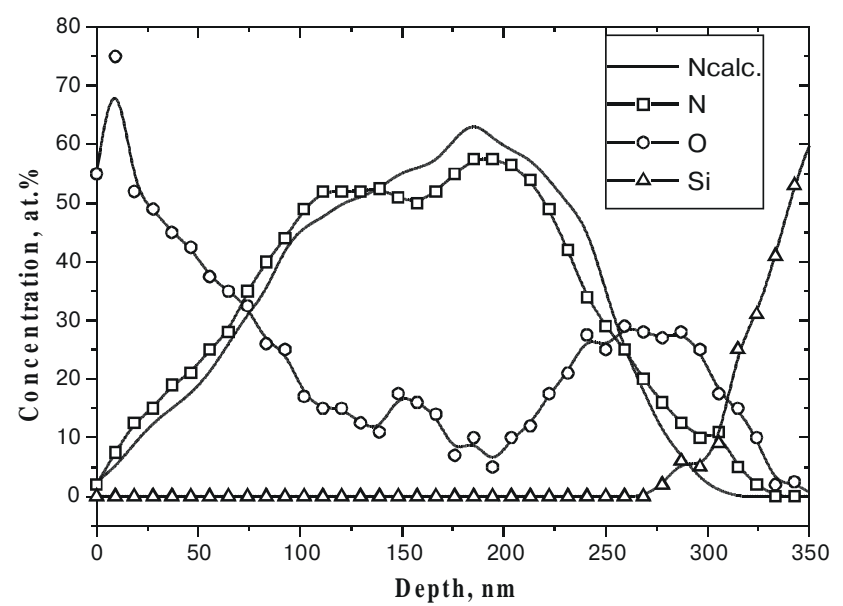

a

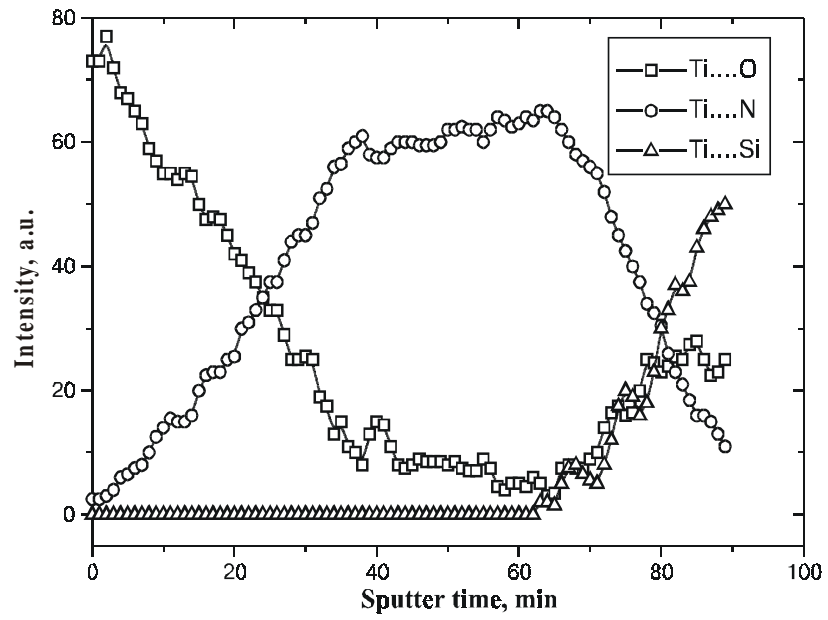

b

Fig.4. $\mathrm{N}^{+}$implantation at $500^{\circ} \mathrm{C}$ into a Ti layer on $\mathrm{Si}\left(E=100 \mathrm{keV}, D=6 \cdot 10^{16} \mathrm{~cm}^{-2}\right)$ : (a): XPS depth profile and calculated ( from AES) nitrogen concentration by means of Eqn. (1), (b): TFA analysis using the PHI-Matlab method.

profile, firstly the oxygen concentration was calculated using the Ti-LMV and 0-KLL peaks. Subsequently, the N concentration was obtained using Eq. (1). Now a much better correlation is obtained between the data for $\mathrm{N}$ in Fig. $4 \mathrm{~b}$ in comparison to that achieved in Fig.2b. The use of Auger spectrometers of another tipe (09 ИОС-005) have given the same results within the deviation no more than 2 at. $\%$.

The application of the PHI-Matlab-software on TIN layers is an other possibility to take into account chemical influences on Auger spectra, which has been demonstrated by Watson et al. [7]. The method involves first applying target factor analysis (TFA) to separate the Auger spectra of $\mathrm{N}$ in $\mathrm{Ti}$ and $\mathrm{TiN}$. Isolation of the spectra of $\mathrm{N}$ and $\mathrm{Ti}$ as TiN begins with Auger spectra recorded from $\mathrm{TiN}_{x}$ samples with different composition. To compare our simple approach with other existing methods of $\mathrm{N}$ quantification we used the TFA analysis, too. The principal component analysis of the Ti spectral data shows that the system now cannot be described by two eigenvectors, a third has to be taken into account. From XPS results this component can be related to $\mathrm{TiO}_{z}$ phase. These three largest eigenvectors were retai-ned to construct the PCA solution while the remaining eigenvectors (representing spectral noise) were discarded. The result is shown in Fig.4b, a good correlation to Fig.4a is found. Therefore, our proposed simple approach can be successfully applied for compositional characterization of $\mathrm{TiN}_{x}$ layers containing oxygen. An interesting technological result, which can be concluded from the depth profile in Fig.4, is the evidence of oxygen out-diffusion from the region where TiN is formed towards the surface and the bulk of the Ti layer.

Fig. 5 shows a typical example of a $0.3 \mu \mathrm{m}$ defect analysis by means of AES in sub-micron TiN structures. As it is seen from the superimposed line-scans, the defect is related to a $\mathrm{TiO}_{z}$ inclusion into the $\mathrm{TiN}$ layer. The quantification procedure of Eq.(1) can easily be used to characterize lateral inhomogenous TiN layer formation in advanced microelectronic structures.

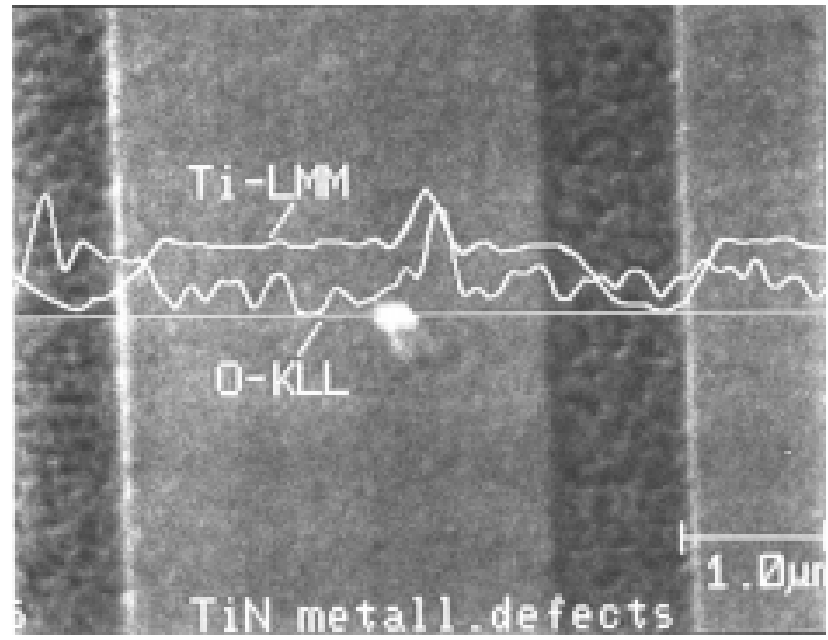

Fig.5. Failure analysis of sub-micron defects in TiN diffusion barriers: AES line scans using Ti-LMM, 0-KLL transitions.

\section{Summary}

A simple empirical method based on Auger peak intensity relations is developed for a quick estimation of TiN, layer composition in semiconductor structures. The results of quantification are found to be in good agreement with XPS profiles and results from Principal Component Analysis (PCA). For defined compositional modification of TiN the layers were implanted using carbon and oxygen ions to study their influence on quantitative analysis. The influence of oxygen was found to be crucial for usual AES analysis but could be taken into account in the proposed method. High carbon concentration shows no significant influence on the $\mathrm{Ti}$ and $\mathrm{N}$ peak shapes. 


\section{Melnik et al.: AES and XPS characterization of TiN layers...}

\section{References}

1. Z. Yu, K. Inagawa, Z. Jin, Tribological Proporties of TiN and TiC Films in Vacuum at High Temperature // Thin solid Films, 264(1), p.p.52 - 58 (1995)

2. T. Hara, A. Yananoue, H. lio, K. Inoue, G. Wahiszu, Properties of Titanium Nitride Films For Barrier Metal in Aluminium Ohmic Contact Systems // Jpn. J. Appl. Phys., 30(7) p.p. 1447 - 1451(1991).

3. P. Yin, S. Maruno,Bias Effect on the Microstructure and Diffusion Barrier Capability of Sputterd TiN and $\mathrm{TiO}_{\mathrm{x}} \mathrm{N}_{\mathrm{y}}$ Films // Jpn. J. Appl. Phys., 31(5A), p.p. 1446 - 1452 (1992).
4. M. Eizenberg, K. Littau, S. Ghanayem, M. Liao, R. Mosely, A. K Sinha, Chemical Vapour Deposited TiCN: a New Barrier Metallization for Submicron Wia and Contact Application // J. Vac. Sci. Techn., A, 13(3), p.p. $590-595$ (1995).

5. Y. Kasukabe, J. Ootubo, S. Nagata, M. Kishimoto, Y. Fujino, S. Yamaguchi, Y.Yamada, TiN Surface Layer Synthesis Using Ion Implantation // Jpn. J. Appl. Phys., 34(7), p.p. 3234 - 3239 (1995).

6. «Metrology Roadmap: A Supplement to the National Technology Roadmap for Semiconductors», SEMATECH, Austin, TX, 1995.

7. D. G. Watson, W.F. Stickle, Physical Electronics Division, Application Note, 8905.

8. Charles Evans \& Assotiates, Application Note AES -AN-004/01, 2/92 